\title{
Special Issue on Tools for Computer Performance Modeling and Reliability Analysis
}

\author{
Giuliano Casale \\ SAP Research \\ CEC Belfast, TEIC Building \\ Shore Road, Newtownabbey \\ BT37 0QB, UK \\ giuliano.casale@sap.com
}

\author{
Richard R. Muntz \\ UCLA \\ Computer Science Dept. \\ 3277A Boelter Hall \\ Los Angeles, CA 90095-1596 \\ muntz@cs.ucla.edu
}

\author{
Giuseppe Serazzi \\ Politecnico di Milano \\ Dip. di Elettr. e Informazione \\ Via Ponzio 34/5 \\ Milano, Italy, 20133 \\ giuseppe.serazzi@polimi.it
}

\section{Editorial Note}

This special issue offers an up-to-date overview on some of the most popular software tools for computer performance modeling and reliability analysis that are currently developed and maintained by research groups in academia. Because of the continuously increasing complexity of computer systems and networks, researchers have developed throughout the years many analytical and simulative approaches to evaluate performance and reliability of realworld systems. However, the mathematical skills required to understand some of these results are often a barrier for their wide-spread adoption. Software tools represent a practical way to overcome this barrier, as they tend to hide most of the mathematical aspects of the modeling or analytical technique used and therefore are more widely accepted.

Although software tools represent an effective way to convey modeling research results into practical applications, we feel that more should be done in advertising to the general public which tools are available and how they differ in terms of functionalities and modeling approaches used. For example, we are not aware of any web page overviewing performance and reliability tools that are made available by academic research groups. Therefore, the chances of a tool of becoming popular seems to be left either to the individual action of the authors or to the popularity of the web sites used to distribute the tool. The eleven invited papers in this special issue try to help in this direction by reviewing the general characteristics of some popular tools for performance and reliability analysis developed in academia. Each paper presents a general description of tool features together with a discussion on upcoming extensions. Table 1 lists in alphabetical order the tools presented in this special issue and overviews some of their main features. For example, the second row lists all tools that can specify and solve a Markov chain, either by analytical methods or by simulation. Indeed, we do not expect to have considered all the tools developed in academia, however we believe that those described in this issue at least represent an important subset of those presently used.

GreatSPN, presented in "The GreatSPN Tool: Recent Enhancements" is a tool specifically tailored to the solution of Generalized Stochastic Petri Nets (GSPNs) and Stochastic Well-Formed Nets (SWNs) with colored tokens and can perform a variety of evaluations such as structural analyses, state-space analysis, simulation, and Markov chain generation. GreatSPN analysis modules can be run either with a graphical user interface or as stand-alone command line programs for batch analysis.

Java Modelling Tools (JMT) is a suite of applications for queueing network analysis and workload characterization. The tool, presented in "JMT Performance Engineering Tools for System modelling" supports simulation of product-form and non-product-form networks, what-if analysis, wizard-based user interaction, and semiautomatic characterization of performance data traces.

The Möbius framework, presented in "Performance and Dependability modeling with Möbius", is a multi-paradigm environment that allows the specification of composite performance and reliability models based on different formalisms such as stochastic activity networks (SANs), fault trees, and the PEPA stochastic process algebra. Möbius simulation traces can be exported in XML to the Traviando visualization tool for model validation and statistical analysis.

The paper "OPEDo: A Tool for the Optimization of Performance and Dependability Models" describes the OPEDo software tool for numerical optimization of performance or dependability metrics in discrete event systems. OPEDo implements local, global, and hybrid search methods which iteratively evaluate the response surface seeking the optimum solution. The tool considers the underlying model as black-box and it interfaces to external applications for model specification and evaluation.

PEPA Eclipse Plug-in, presented in "The PEPA Eclipse Plugin" supports the definition of stochastic process algebra models based on the Performance Evaluation Process Algebra (PEPA) language. PEPA Eclipse Plug-in is centered around the Pepato library for static, Markovian, and ODE analysis of PEPA models by means of numerical and simulative methods. The tool runs inside the open source Eclipse workspace and supports seamless upgrade to newer versions via the Eclipse interface.

PIPE2, described in "PIPE2: A Tool for the Performance Evaluation of Generalised Stochastic Petri Nets" is a tool for the analysis of Generalized Stochastic Petri Nets (GSPNs). The application supports structural and performance analysis modules for evaluation of GSPNs. PIPE2 integrates an original formalism for graphical specification of performance queries and adopts a client-server software architecture that allows for parallel or distributed model analysis.

The PRISM tool described in the paper "PRISM: Probabilistic Model Checking for Performance and Reliability Analysis" is a probabilistic model checker that supports Markov chains, Markov decision processes, and reward models. PRISM includes a language for textual modeling and allows for the validation of model properties based on a temporal logic approach. Evaluation methods include simulation, numerical and graph-theoretical model checking, and symbolic techniques based on binary decision diagrams.

The QPME tool described in "QPME A Performance Modeling Tool Based on Queueing Petri Nets" supports the specification of Queueing Petri Nets (QPNs), a formalism that integrates queueing places within stochastic Petri nets. The tool allows the evaluation of QPNs by Monte carlo simulations and has native support for 


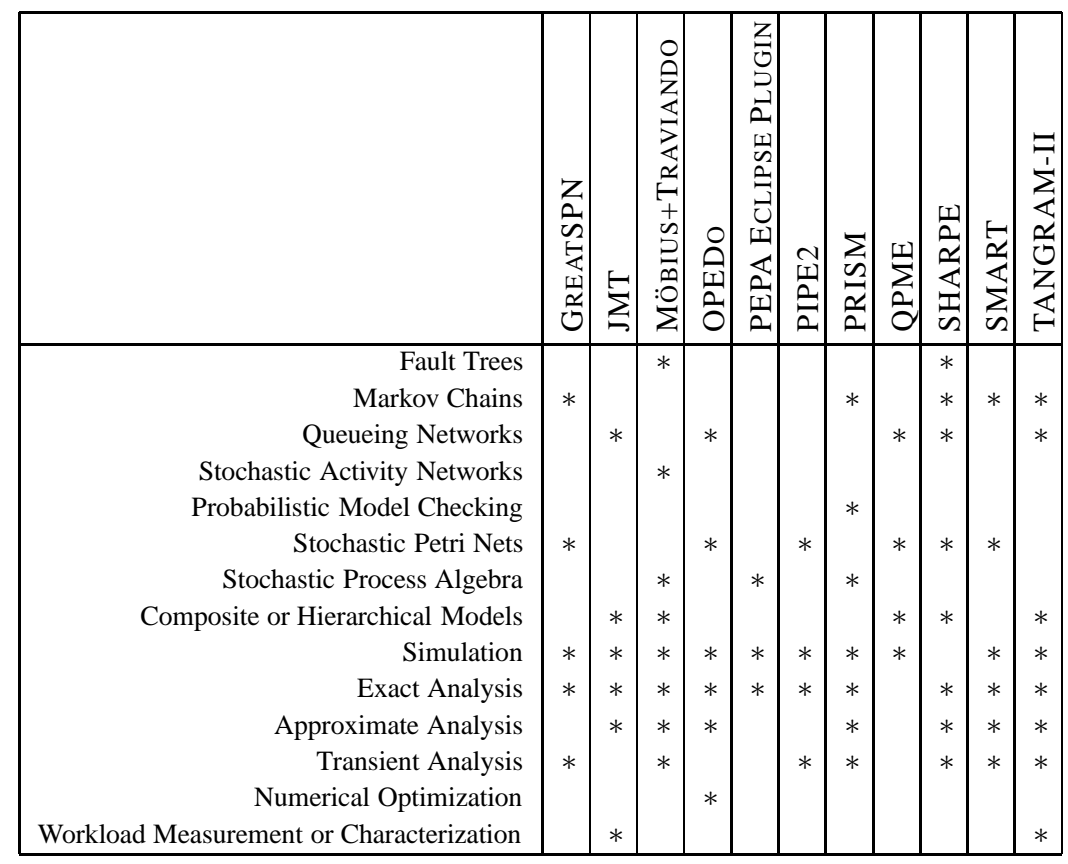

Table 1: Main features of performance and reliability tools presented in the special issue

colored tokens, incidence functions, and several performance data collection modes. QPME also includes the QPE editor which allows graphical specification of model structure.

SHARPE is a tool for semi-symbolic analysis of fault trees, reliability block diagrams, acyclic series-parallel graphs, acyclic and cyclic Markov and semi-Markov models, GSPNs, and product-form queueing networks. In "SHARPE at the Age of Twenty Two" the development of the tool is described together with an overview of advanced features such as exact model decomposition, exponomial distributions and their practical application to real-world availability models.

The SMART tool presented in "Advanced features in SMART: the Stochastic Model checking Analyzer for Reliability and Timing" is a software package for model-checking and for numerical and simulative evaluation of stochastic models which are an extended form of Petri nets. SMART uses a compact representation of large scale models based on different types of decision diagrams which can be evaluated either exactly or approximately for increased computational efficiency.

TANGRAM-II, described in the paper "The TANGRAM-II Integrated Modeling Environment for Computer Systems and Networks" is a comprehensive suite of tools for system modeling and traffic measurement. The analytical solution engine supports both Markovian and non-Markovian models; the simulation engine can also evaluate fluid-models. TANGRAM-II also supports Hybrid Markov Models (HMMs) and integrates a network measurement engine.

In conclusion, this special issue represents an effort in the direction of increasing the visibility of tools for performance and reliability modeling developed within the SIGMETRICS community. We sincerely thank all authors for their extensive work in the preparation of the papers. We also thanks Evgenia Smirni for her support throughout the phases of development of this special issue.

Giuliano Casale Richard R. Muntz Giuseppe Serazzi 